\title{
$\begin{array}{ll}\text { Research Square } & \begin{array}{l}\text { Preprints are preliminary reports that have not undergone peer review. } \\ \text { They should not be considered conclusive, used to inform clinical practice, } \\ \text { or referenced by the media as validated information. }\end{array}\end{array}$
}

\section{Associations of residential greenness with hypertension and blood pressure in a Chinese rural population: A cross-sectional study}

Jie Jiang

Department of Global Health, School of Health Sciences, Wuhan University

\section{Gongbo Chen}

Guangdong Provincial Engineering Technology Research Center of Environmental and Health Risk Assessment, Department of Occupational and Environmental Health, School of Public Health, Sun Yatsen University

\section{Baojing Li}

Department of Global Public Health, Karolinska Institute

$\mathrm{Na} \mathrm{Li}$

Department of Global Health, School of Health Sciences, Wuhan University, Wuhan

\section{Feifei Liu}

Department of Global Health, School of Health Sciences, Wuhan University

\section{Yuanan Lu}

Environmental Health Laboratory, Department of Public Health Sciences, University of hawii at Manoa

\section{Yuming Guo}

Department of Epidemiology and Preventive Medicine, School of Public Health and Preventive Medicine, Monash University

\section{Shanshan Li}

Department of Epidemiology and Preventive Medicine, School of Public Health and Preventive Medicine, Monash University

\section{Lifeng Chen}

Department of laboratory medicine, Wuhan Pulmonary Hospital

Hao Xiang ( $\nabla$ xianghao@whu.edu.cn )

Department of Global Health, School of Health Science, Wuhan University

\section{Research Article}

Keywords: Greenness, Hypertension, Blood pressure, Rural population

Posted Date: April 1st, 2021

DOl: https://doi.org/10.21203/rs.3.rs-172790/v1 
License: (c) (i) This work is licensed under a Creative Commons Attribution 4.0 International License. Read Full License 


\section{Abstract}

Background: Limited epidemiological literature identified the associations between residential greenness and hypertension in low/middle-income countries.

Methods: A random sampling strategy was adopted to recruit 39259 residents, $\geq 18$ years, and from 5 counties in central China. Blood pressure was measured based on the protocol of the American Heart Association. Hypertension was defined according to the 2010 Chinese guidelines for the management of hypertension. The satellite-derived Normalized Difference Vegetation Index (NDVI) and Enhanced Vegetation Index (EVI) were applied to estimate the residential greenness. Mixed logit model and mixed linear model were utilized to explore the relationships of residential greenness with hypertension and blood pressure.

Results: High residential greenness was associated with lower odds of hypertension and blood pressure levels. For instance, an interquartile range (IQR) increase in $\mathrm{NDVI}_{500 \mathrm{~m}}$ was linked with lower odds of hypertension ( $\mathrm{OR}=0.92,95 \% \mathrm{Cl} 0.88$ to 0.95$)$, a decrease of $-0.88 \mathrm{~mm} \mathrm{Hg}(95 \% \mathrm{Cl}-1.17$ to -0.58$)$ and -0.64 $\mathrm{mm} \mathrm{Hg}(95 \% \mathrm{Cl}-0.82$ to -0.46$)$ in SBP and DBP, respectively. The effect of residential greenness was more pronounced in males, smokers, and drinkers.

Conclusions: Long-term exposure to residential greenness may reduce the risk of hypertension. More prospective studies are needed to verify the hypothesis.

\section{Introduction}

Cardiovascular diseases (CVDs) drive huge deaths worldwide and hypertension is the major cause of CVDs (GBD 2018). In 2017, over 10 million global deaths were due to high systolic blood pressure (GBD 2018). Along with rapid urbanization, the hypertension prevalence was increasing, reflecting inadequate control in China (Lu et al. 2017). Apart from hereditary, behavioral, and other recognized hypertensionrelated factors, environmental impacts have been demonstrated in the published studies (Vienneau et al. 2017). The underlying greenspace-health mechanisms include weakening injury (eg, air pollutionbuffering, noise-buffering, heat-buffering), capability recovery (eg, alleviating anxiety and depression), and improving capability (eg, offering breathing spaces, promoting social-interaction activities) (Markevych et al. 2017).

Previous studies have illustrated the associations between residential greenness and hypertension in Europe (Bijnens et al. 2017; Bloemsma et al. 2019; de Keijzer et al. 2019; Groenewegen et al. 2018; Jendrossek et al. 2017; Madhloum et al. 2019; Markevych et al. 2014), the United States (Brown et al. 2016), Australia (Dzhambov et al. 2018), and several low/middle-income countries (Jia et al. 2018; Lane et al. 2017; Moreira et al. 2020; Xiao et al. 2020; Yang et al. 2019). However, the results of those analyses remained mixed. For instance, Dzhambov et al. (2018) revealed a 36\% lower hypertension prevalence per interquartile range (IQR) increase in Normalized difference vegetation index $\left(\mathrm{NDVI}_{500 \mathrm{~m}}\right)$ in adults. Similar relationships were detected in the newborns (Madhloum et al. 2019) and children (Xiao et al. 2020). In 
addition, null associations were identified in several studies (Bloemsma et al. 2019; Jendrossek et al. 2017; Moreira et al. 2020). For example, Jendrossek et al. (2017) concluded odds ratio (OR) $=0.889$ $(95 \% \mathrm{Cl} 0.561-1.409)$. Furthermore, the majority of published studies were carried out in developed countries (Jia et al. 2018; Lane et al. 2017). Convincing conclusions, undoubtedly, demands extensive investigation in various populations. Here, this study performed the population-based analyses in Chinese rural dwellers, therefore, providing new evidence in low/middle-income countries.

We hypothesized that high residential greenness may reduce the risk of hypertension. Also, the potential effect modifiers in the relationships were examined.

\section{Methods}

\section{Study population}

Rural Cohort design was described in the prior study (ChiCTR-O0C-15006699) (Liu et al. 2019b). Briefly, a random sampling was applied to select permanent dwellers, 18-79 years, and living in 5 rural zones in central China (Fig 1 and 2). Overall, 39259 participated in the study. Subjects without outcome (eg, SBP/DBP) were excluded in the final analyses. The signed informed consent was received before the survey. This study was approved by the Zhengzhou University review board.

\section{Outcome}

SBP/DBP was measured thrice utilizing OMRON HEM-770A according to the American Heart Association guideline (Pickering et al. 2005). The protocol was published previously (Li et al. 2020). Prior to measurement, smoking, drinking, and physical exercise were forbidden within half an hour. In addition, talking was forbidden during the measurement. Hypertension, by definition of 2010 Chinese guideline (Wang et al. 2018), SBP $\geq 140 \mathrm{~mm} \mathrm{Hg}, \mathrm{DBP} \geq 90 \mathrm{~mm} \mathrm{Hg}$, diagnosis history of hypertension, and use of the antihypertensive drug.

\section{Greenness exposure}

The NDVI and EVI databases were downloaded from the National Aeronautics and Space Administration to estimate the residential greenness for each participant. The assessment was described elsewhere (Xie et al. 2020). The two vegetation indexes could effectively characterize vegetation states and processes. NDVI has been used widely for environmental applications (Jia et al. 2018). Compared with NDVI, EVI could minimize canopy-soil variations and improved sensitivity over dense vegetation conditions (Qiu et al. 2018). Long-term exposure to greenness was defined as the 3-year average of NDVI/EVI prior to the survey.

\section{Covariates}

Demographics included age, sex, region (Xinxiang, Suiping, Yuzhou, Yima, and Tongxu), marriage (no, yes), education (low, medium, high), income/month (yuan) $(\leq 500,500 \sim, 1000)$. Behavioral factors 
included smoking, drinking, fat-rich diet, and exercise. Fat-rich diet (Yang et al. 2018) and exercise (Craig et al. 2003) information were obtained from a standard questionnaire. Health status included family history of hypertension and body mass index (BMI). A spatiotemporal model was performed to assess the exposure to fine particulate matter $\left(\mathrm{PM}_{2.5}\right)$ at a $0.1^{\circ} \times 0.1^{\circ}$ spatial resolution (Chen et al. 2018). The accuracy of the method was verified elsewhere (10-fold cross-validation $R^{2}=86 \%$ ) (Liu et al. 2019a).

\section{Statistical analysis}

Mixed logit model and mixed linear model were employed to explore the associations between residential greenness and hypertension and blood pressure. Long-term exposure to greenness was utilized in the main model. The models were widely used in recent studies (Xiao et al. 2020; Yang et al. 2019). Given the possible influence of regional clustering, the region was controlled as a random effect in the analyses (Xie et al. 2020). Adjustment of fixed effects was as follows: (1) Crude model: non; (2) Adjusted model: adjusted for age, sex, marriage, education, income/month, smoking, drinking, fat-rich diet, exercise, family history of hypertension, BMI, and $\mathrm{PM}_{2.5}$ (Dzhambov et al. 2018; Lane et al. 2017). To test effect modifications of behavioral factors in the greenness-hypertension/blood pressure associations, the interaction analysis was performed by putting the interaction items in the adjusted model.

Considering the possible mediation in the greenness-hypertension/blood pressure pathways (eg, air pollution, exercise) (de Keijzer et al. 2019; Xie et al. 2020), serial mediation analyses were carried out. To verify the reliability of conclusions, sensitivity analyses were conducted: (1) The average of 1-year and 2year $\mathrm{NDVI}_{500 \mathrm{~m}} / \mathrm{EVI}_{500 \mathrm{~m}}$ prior to the survey was employed to test long-term impacts of greenness on hypertension and blood pressure; (2) The greenness-hypertension/blood pressure relationships were investigated utilizing different buffers ( $\mathrm{NDVI}_{1000 \mathrm{~m}} / \mathrm{EVI}_{1000 \mathrm{~m}}, \mathrm{NDVI}_{3000 \mathrm{~m}} / \mathrm{EVI}_{3000 \mathrm{~m}}$ ); (3) To eliminate the causal effect of hypertension on blood pressure, subjects with hypertension and taking the antihypertensive drug were excluded.

Analyses were conducted in R 4.0.2 loading "Ime4" and "mediation" packages.

\section{Results}

This analysis involved 39094 participants (60.6\% females, $n=23$ 677) (Table 1). The characteristics of participants by region was presented in Table S1. Among 39094 participants, $19.1 \%(n=7458)$ were smokers, $18.0 \%(n=7053)$ were drinkers, $19.1 \%(n=7461)$ had a fat-rich t diet, and 32.3\% $(n=12614)$ reported a low level of physical exercise. The 3-year average $\mathrm{NDVI}_{500 \mathrm{~m}}$ and $\mathrm{EVI}_{500 \mathrm{~m}}$ were 0.4785 and 0.3380 units (Table S2). A total of 12763 hypertensive patients were identified (prevalence $=32.6 \%$ ), $61.3 \%$ were self-reported, $38.7 \%$ were diagnosed by trained physicians. 
Table 1

Characteristics of participants.

\begin{tabular}{|c|c|c|c|c|}
\hline \multicolumn{2}{|l|}{ Variables } & Hypertension & Non-hypertension & All \\
\hline \multicolumn{2}{|l|}{$\mathrm{N}$} & 12763 & 26331 & 39094 \\
\hline \multicolumn{2}{|l|}{ Age $($ mean $\pm S D)$} & $60.37 \pm 10.09$ & $53.26 \pm 12.43$ & $55.58 \pm 12.18$ \\
\hline \multirow[t]{2}{*}{$\operatorname{Sex}(n, \%)$} & Male & $5094(39.9)$ & $10323(39.2)$ & 15417 (39.4) \\
\hline & Female & 7669 (60.1) & $16008(60.8)$ & 23677 (60.6) \\
\hline \multirow[t]{3}{*}{ Education (n, \%) } & Low & $6793(53.2)$ & 10689 (40.6) & 17482 (44.7) \\
\hline & Medium & 4395 (34.4) & $11197(42.5)$ & 15592 (39.9) \\
\hline & High & 1575 (12.3) & 4445 (16.9) & $6020(15.4)$ \\
\hline \multicolumn{2}{|l|}{ Married (n, \%) } & $11121(87.1)$ & 23984 (91.1) & 35105 (89.8) \\
\hline \multirow[t]{3}{*}{ Income/month (n, \%) } & $\leq 500$ yuan & 5045 (39.5) & 8899 (33.8) & 13944 (35.7) \\
\hline & $500 \sim$ & $4200(32.9)$ & $8650(32.9)$ & 12850 (32.9) \\
\hline & $\geq 1000$ yuan & $3518(27.6)$ & $8782(33.4)$ & $12300(31.4)$ \\
\hline \multirow[t]{3}{*}{ Smoking (n, \%) } & Never & $9353(73.3)$ & 19109 (72.6) & $28462(72.8)$ \\
\hline & Former & $1333(10.4)$ & $1841(7.0)$ & $1819(4.7)$ \\
\hline & Current & 2077 (16.3) & $5381(20.4)$ & 7458 (19.1) \\
\hline \multirow[t]{3}{*}{ Drinking (n, \%) } & Never & 9765 (76.5) & 20457 (77.7) & $30222(77.3)$ \\
\hline & Former & $709(5.6)$ & $1110(4.2)$ & $1819(4.7)$ \\
\hline & Current & $2289(17.9)$ & $4764(18.1)$ & 7053 (18.0) \\
\hline \multirow[t]{3}{*}{ Exercise (n, \%) } & Low & $4783(37.5)$ & 7831 (29.7) & $12614(32.3)$ \\
\hline & Medium & 4377 (34.3) & $10391(39.5)$ & $14768(37.8)$ \\
\hline & High & $3603(28.2)$ & 8109 (30.8) & $11712(29.9)$ \\
\hline \multicolumn{2}{|l|}{ Fat-rich diet (n, \%) } & 2009 (15.7) & $5452(20.7)$ & $7461(19.1)$ \\
\hline \multicolumn{2}{|c|}{ Family history of hypertension (n, \%) } & $3515(27.5)$ & $4049(15.4)$ & $7564(19.3)$ \\
\hline \multicolumn{2}{|c|}{ Body mass index $\left(\mathrm{kg} / \mathrm{m}^{2}\right.$, mean $\left.\pm \mathrm{SD}\right)$} & $26.03 \pm 3.65$ & $24.25 \pm 3.36$ & $24.83 \pm 3.56$ \\
\hline \multicolumn{2}{|c|}{$\mathrm{PM}_{2.5}\left(\mu \mathrm{g} / \mathrm{m}^{3}\right.$, mean $\left.\pm \mathrm{SD}\right)$} & $73.86 \pm 2.43$ & $73.21 \pm 2.61$ & $73.42 \pm 2.57$ \\
\hline
\end{tabular}

Abbreviations: SD, standard deviation; $\mathrm{PM}_{2.5}$, fine particulate matter. 
High residential greenness was related to $8 \%-11 \%$ lower hypertension prevalence, $0.79-1.18 \mathrm{mmHg}$ decreased SBP, and 0.64-1.11 mm Hg decreased DBP (Fig. 3). For example, each IQR (0.08 units) increase in $\mathrm{NDVI}_{500 \mathrm{~m}}$ was related to $8 \%$ lower odds of hypertension, a reduction of $0.88 \mathrm{mmHg}$ in SBP, and $0.64 \mathrm{mmHg}$ in DBP in the adjusted model. Interaction analysis concluded that the effect of residential greenness on hypertension/blood pressure was more pronounced in males, smokers, and drinkers $\left(P_{\text {interaction }}<0.05\right)$ (Fig. 4). For instance, high $\mathrm{NDVI}_{500 \mathrm{~m}}$ was related to $13 \%$ lower odds of hypertension among males, whereas $5 \%$ among females $\left(P_{\text {interaction }}=0.005\right)$. In addition, a decrease of $1.30 \mathrm{~mm} \mathrm{Hg}$ in SBP among males, compared with $0.63 \mathrm{mmHg}$ among females $\left(P_{\text {interaction }}=0.002\right)$.

$\mathrm{PM}_{2.5}$, exercise, and BMI partially mediated the relationships between residential greenness and hypertension and blood pressure (Table S3). The proportion of mediation for $\mathrm{PM}_{2.5}$, exercise, and $\mathrm{BMI}$ varied between $3.7-21.6 \%, 0.7-4.4 \%$, and $23.6-36.7 \%$, respectively. Compared with main analyses, using the 1-year/2-year average $\mathrm{NDVI}_{500 \mathrm{~m}} / \mathrm{EVI}_{500 \mathrm{~m}}$ and $\mathrm{NDVI}_{1000 \mathrm{~m}} / \mathrm{EVI}_{1000 \mathrm{~m}}$ showed similar results (Fig S1). The enhanced association was detected using $\mathrm{NDVI}_{3000 \mathrm{~m}} / \mathrm{EVI}_{3000 \mathrm{~m}}$. For example, each IQR increase in $\mathrm{NDVI}_{3000 \mathrm{~m}}$ was related to $19 \%$ lower odds of hypertension. Changes in SBP/DBP remained similar when participants taking the antihypertensive drug were excluded. After excluding hypertensive patients, the relationships were attenuated but remained significant. Each IQR increase in $\mathrm{NDVI}_{500 \mathrm{~m}}$ was linked to 0.40 $\mathrm{mmHg}$ lower SBP and $0.32 \mathrm{~mm} \mathrm{Hg}$ lower DBP.

\section{Discussion}

The studies of the greenness-hypertension pathways are still in the initial stage and lack of systematic studies in low/middle-income countries. We found that elevated residential greenness was associated with $8 \%-11 \%$ decreased hypertension prevalence, $0.79-1.18 \mathrm{mmHg}$ lower SBP, and 0.64-1.11 mm Hg lower DBP. In addition, sex, smoking, and drinking could further modify the relationships. Given increasing environmental pollution accompanied by the accelerated urbanization process and high hypertension prevalence in China as well as other low/middle-income countries, our findings may be particularly important for public health.

In the present study, each IQR increment in $\mathrm{NDVI}_{500 \mathrm{~m}}$ was related to $8 \%$ lower odds of hypertension. Similar conclusions were drawn not only in adults (Brown et al. 2016; de Keijzer et al. 2019; Dzhambov et al. 2018; Jia et al. 2018; Vienneau et al. 2017; Yang et al. 2019) but also in children (Xiao et al. 2020). For instance, Brown et al. (2016) indicated 13\% lower odds of hypertension among elderly Americans ( $\geq 65$ years) attributed to high residential greenness. Additionally, each 0.1-unit increment in $\mathrm{NDVI}_{500 \mathrm{~m}} \mathrm{Was}$ related to $24 \%$ lower hypertension prevalence in Chinese children (Xiao et al. 2020). However, Jendrossek et al. (2017) revealed a null association among German adults (OR $=0.889,95 \% \mathrm{Cl} 0.561-1.409) \mathrm{A}$ metaanalysis of 4 studies concluded no significant greenness-hypertension association $(O R=0.99,95 \% \mathrm{Cl}$ 0.81-1.20) (Twohig-Bennett and Jones 2018). Limited evidence from low/middle-income countries, therefore, a comprehensive greenness-hypertension pathway remained deeply studied. In addition, the meta-analysis identifying those relationships need to be updated. 
High residential greenness was linked with lower SBP/DBP in the current analysis. In line with our result, Lane et al. (2017) found $4.3 \mathrm{mmHg}$ lower SBP and $1.2 \mathrm{mmHg}$ lower DBP per IQR increment in $\mathrm{NDVI}_{250 \mathrm{~m}}$ among Indian adults. However, inconsistent results existed in several previous studies (Bijnens et al. 2017; Bloemsma et al. 2019; Dzhambov et al. 2018; Madhloum et al. 2019; Markevych et al. 2014; Xiao et al. 2020; Yang et al. 2019). For instance, the significant $\mathrm{NDVI}_{500 \mathrm{~m}}$-SBP association was observed, whereas the null NDVI $500 \mathrm{~m}$-DBP association in Chinese adults (Yang et al. 2019). In addition, Madhloum et al. (2019) drew the opposite conclusion indicating no significant greenness-SBP relationship, whereas the significant greenness-DBP relationship in newborns. No significant association was found between greenness and SBP/DBP in adolescents (Bloemsma et al. 2019). The study population mentioned above (eg, adult, newborn, children) have different characteristics that may modify the effect of greenness. Besides, differences in study design, greenness assessment may explain the inconsistency. Thus, the greenness-SBP/DBP pathways remained unclear.

The mechanism by which long-term exposure to residential greenness reduces the risk of hypertension could be explained by immunological pathways (Rook 2013). Specifically, people residing in green space are exposed to more diverse microbes beneficial to the host immune system (improving immune regulation) and less noise and air pollution (reducing inflammatory response), are more likely to increase physical activity (strengthening immune and nervous system), promote the exchange of microbiota (increasing social interactions), and promote metabolism (sunlight helping in the synthesis of vitamin D). Also, high residential greenness ease tension and depression by increasing social interactions (Banay et al. 2019).

Interaction analysis showed that males, smokers, and drinkers tended to be more susceptible to the effect of greenness. Whether sex modifies the greenness-hypertension relationship remained unclear in previous studies (Dzhambov et al. 2018; Jia et al. 2018; Xiao et al. 2020; Yang et al. 2019). For example, Jia et al. (2018) reported $\mathrm{OR}_{\text {male }}=0.14(95 \% \mathrm{Cl} 0.11-0.48)$ and $\mathrm{OR}_{\text {female }}=0.45(95 \% \mathrm{Cl} 0.36-0.63)$ and it was consistent with our finding. However, Yang et al. (2019) found a null association in males, whereas the a significant association in females. In addition, Xiao et al. (2020) observed no significant sex interaction in $\mathrm{NDVI}_{500 \mathrm{~m}}$-hypertension relationship $\left(\mathrm{OR}_{\text {male }}=0.81 \mathrm{vs} . \mathrm{OR}_{\text {female }}=0.72, P_{\text {interaction }}=0.279\right)$. Smoking and drinking could trigger inflammatory response, oxidative stress, and metabolic disorders, which further affect systemic vascular resistance (Joseph et al. 2017). Long-term exposure to greenness could provide diverse microbes, some of which are important inducers of the immunoregulatory pathways, and activation of the immune regulatory system consequently reduced chronic inflammation (Rook 2013). Thus, the effect of greenness on hypertension and blood pressure was more pronounced in smokers and drinkers.

Several limitations existed in the present study. First, as an inherent drawback of cross-sectional design, causal associations should be treated with caution. Second, demographics, behavioral factors, and health status were collected using the questionnaire, which may introduce recall bias. Third, some 
potential confounders including noise, the walkability of the community, and indoor exposure to greenness were not adjusted because of unavailability (Dzhambov et al. 2018; Xiao et al. 2020).

\section{Conclusion}

Long-term exposure to residential greenness may reduce the risk of hypertension. Furthermore, males, smokers, and drinkers were more susceptible to the effect of greenness. Given the limitations of design, prospective studies are warranted in future analysis.

\section{Declarations}

\section{Acknowledgments}

We acknowledge the participants in the study.

\section{Authors' contributions}

JJ: analysis, visualization, writing manuscript; GC: methodology, interpretation; BL: interpretation; NL: analysis, data curation; FL: validation, data curation; YL: methodology, supervision; YG: validation, funding acquisition; SL: methodology, funding acquisition; LC: visualization, methodology; HX: editing, funding acquisition. All authors read and approved the final manuscript.

\section{Funding}

Our work was funded by the National Natural Science Foundation of China (81903279), the Bill \& Melinda Gates Foundation (OOP1148464), and the Hubei Province Natural Science Foundation (2018CFB634). Dr. Guo was supported by the Career Development Fellowship of the Australian National Health and Medical Research Council (ANHMRC) (APP1107107). Dr. S. Li was supported by the Early Career Fellowship of ANHMRC (APP1109193).

\section{Ethics approval and consent to participate}

This study was approved by the Zhengzhou University review board.

\section{Consent for publication}

Not applicable

\section{Data availability}

Upon reasonable request, data are available from the corresponding author.

\section{Competing interests}

No competing interests 


\section{References}

Banay RF et al. (2019) Greenness and Depression Incidence among Older Women Environmental Health Perspectives 127 doi:10.1289/ehp1229

Bijnens EM, Nawrot TS, Loos RJ, Gielen M, Vlietinck R, Derom C, Zeegers MP (2017) Blood pressure in young adulthood and residential greenness in the early-life environment of twins Environ Health 16:53 doi:10.1186/s12940-017-0266-9

Bloemsma LD et al. (2019) Green space, air pollution, traffic noise and cardiometabolic health in adolescents: The PIAMA birth cohort Environ Int 131:104991 doi:10.1016/j.envint.2019.104991

Brown SC et al. (2016) Neighborhood Greenness and Chronic Health Conditions in Medicare Beneficiaries American Journal of Preventive Medicine 51:78-89 doi:10.1016/j.amepre.2016.02.008

Chen $\mathrm{G}$ et al. (2018) A machine learning method to estimate PM2.5 concentrations across China with remote sensing, meteorological and land use information Sci Total Environ 636:52-60 doi:10.1016/j.scitotenv.2018.04.251

Craig CL et al. (2003) International physical activity questionnaire: 12-country reliability and validity Med Sci Sports Exerc 35:1381-1395 doi:10.1249/01.MSS.0000078924.61453.FB

de Keijzer $\mathrm{C}$ et al. (2019) Long-term exposure to greenspace and metabolic syndrome: A Whitehall II study Environ Pollut 255:113231 doi:10.1016/j.envpol.2019.113231

Dzhambov AM, Markevych I, Lercher P (2018) Greenspace seems protective of both high and low blood pressure among residents of an Alpine valley Environ Int 121:443-452 doi:10.1016/j.envint.2018.09.044

GBD (2018) Global, regional, and national comparative risk assessment of 84 behavioural, environmental and occupational, and metabolic risks or clusters of risks for 195 countries and territories, 1990-2017: a systematic analysis for the Global Burden of Disease Study 2017 Lancet 392:1923-1994 doi:10.1016/s0140-6736(18)32225-6

Groenewegen PP et al. (2018) Neighbourhood social and physical environment and general practitioner assessed morbidity Health Place 49:68-84 doi:10.1016/j.healthplace.2017.11.006

Jendrossek M et al. (2017) Residential Air Pollution, Road Traffic, Greenness and Maternal Hypertension: Results from GINIplus and LISAplus Int J Occup Environ Med 8:131-142 doi:10.15171/ijoem.2017.1073

Jia X et al. (2018) Cardiovascular diseases in middle aged and older adults in China: the joint effects and mediation of different types of physical exercise and neighborhood greenness and walkability Environmental Research 167:175-183 doi:10.1016/j.envres.2018.07.003 
Joseph P et al. (2017) Reducing the Global Burden of Cardiovascular Disease, Part 1: The Epidemiology and Risk Factors Circ Res 121:677-694 doi:10.1161/CIRCRESAHA.117.308903

Lane KJ, Stokes EC, Seto KC, Thanikachalam S, Thanikachalam M, Bell ML (2017) Associations between Greenness, Impervious Surface Area, and Nighttime Lights on Biomarkers of Vascular Aging in Chennai, India Environ Health Perspect 125:087003 doi:10.1289/EHP541

Li N et al. (2020) Associations between long-term exposure to air pollution and blood pressure and effect modifications by behavioral factors Environmental Research 182 doi:10.1016/j.envres.2019.109109

Liu F et al. (2019a) Associations of long-term exposure to PM1, PM2.5, NO2 with type 2 diabetes mellitus prevalence and fasting blood glucose levels in Chinese rural populations Environ Int 133:105213 doi:10.1016/j.envint.2019.105213

Liu X et al. (2019b) Cohort Profile: The Henan Rural Cohort: a prospective study of chronic noncommunicable diseases Int J Epidemiol 48:1756-1756j doi:10.1093/ije/dyz039

Lu J et al. (2017) Prevalence, awareness, treatment, and control of hypertension in China: data from 1.7 million adults in a population-based screening study (China PEACE Million Persons Project) Lancet 390:2549-2558 doi:10.1016/s0140-6736(17)32478-9

Madhloum $\mathrm{N}$ et al. (2019) Neonatal blood pressure in association with prenatal air pollution exposure, traffic, and land use indicators: An ENVIRONAGE birth cohort study Environ Int 130:104853 doi:10.1016/j.envint.2019.05.047

Markevych I et al. (2017) Exploring pathways linking greenspace to health: Theoretical and methodological guidance Environ Res 158:301-317 doi:10.1016/j.envres.2017.06.028

Markevych I et al. (2014) A cross-sectional analysis of the effects of residential greenness on blood pressure in 10-year old children: results from the GINIplus and LISAplus studies BMC Public Health 14:477 doi:10.1186/1471-2458-14-477

Moreira TCL, Polizel JL, Santos IdS, Silva Filho DF, Bensenor I, Lotufo PA, Mauad T (2020) Green Spaces, Land Cover, Street Trees and Hypertension in the Megacity of São Paulo International Journal of Environmental Research and Public Health 17 doi:10.3390/ijerph17030725

Pickering TG et al. (2005) Recommendations for blood pressure measurement in humans and experimental animals: part 1: blood pressure measurement in humans: a statement for professionals from the Subcommittee of Professional and Public Education of the American Heart Association Council on High Blood Pressure Research Circulation 111:697-716 doi:10.1161/01.CIR.0000154900.76284.F6

Qiu J, Yang J, Wang Y, Su H (2018) A comparison of NDVI and EVI in the DisTrad model for thermal subpixel mapping in densely vegetated areas: a case study in Southern China International Journal of Remote Sensing 39:2105-2118 doi:10.1080/01431161.2017.1420929 
Rook GA (2013) Regulation of the immune system by biodiversity from the natural environment: an ecosystem service essential to health Proc Natl Acad Sci U S A 110:18360-18367 doi:10.1073/pnas.1313731110

Twohig-Bennett C, Jones A (2018) The health benefits of the great outdoors: A systematic review and meta-analysis of greenspace exposure and health outcomes Environ Res 166:628-637 doi:10.1016/j.envres.2018.06.030

Vienneau D, de Hoogh K, Faeh D, Kaufmann M, Wunderli JM, Roosli M, Group SNCS (2017) More than clean air and tranquillity: Residential green is independently associated with decreasing mortality Environ Int 108:176-184 doi:10.1016/j.envint.2017.08.012

Wang Z et al. (2018) Status of Hypertension in China: Results From the China Hypertension Survey, 20122015 Circulation 137:2344-2356 doi:10.1161/circulationaha.117.032380

Xiao X et al. (2020) Greenness around schools associated with lower risk of hypertension among children: Findings from the Seven Northeastern Cities Study in China Environ Pollut 256:113422 doi:10.1016/j.envpol.2019.113422

Xie Y et al. (2020) Association between residential greenness and sleep quality in Chinese rural population Environment International 145:106100 doi:https://doi.org/10.1016/j.envint.2020.106100 Yang BY et al. (2019) Community greenness, blood pressure, and hypertension in urban dwellers: The 33 Communities Chinese Health Study Environ Int 126:727-734 doi:10.1016/j.envint.2019.02.068

Yang YX et al. (2018) New Chinese dietary guidelines: healthy eating patterns and food-based dietary recommendations Asia Pac J Clin Nutr 27:908-913 doi:10.6133/apjcn.072018.03

\section{Figures}


The permanent residents aged 18-79 years in China (July 2015 - September 2017)

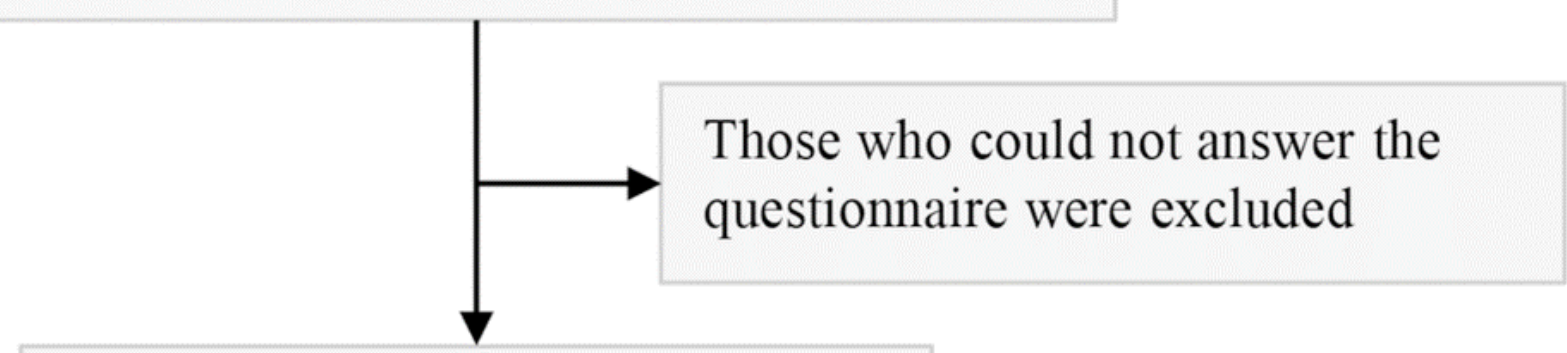

5 rural counties in Henan province

$\mathbf{1}$ to $\mathbf{3}$ communities in each county were selected by the local Centre for Disease Control and Prevention

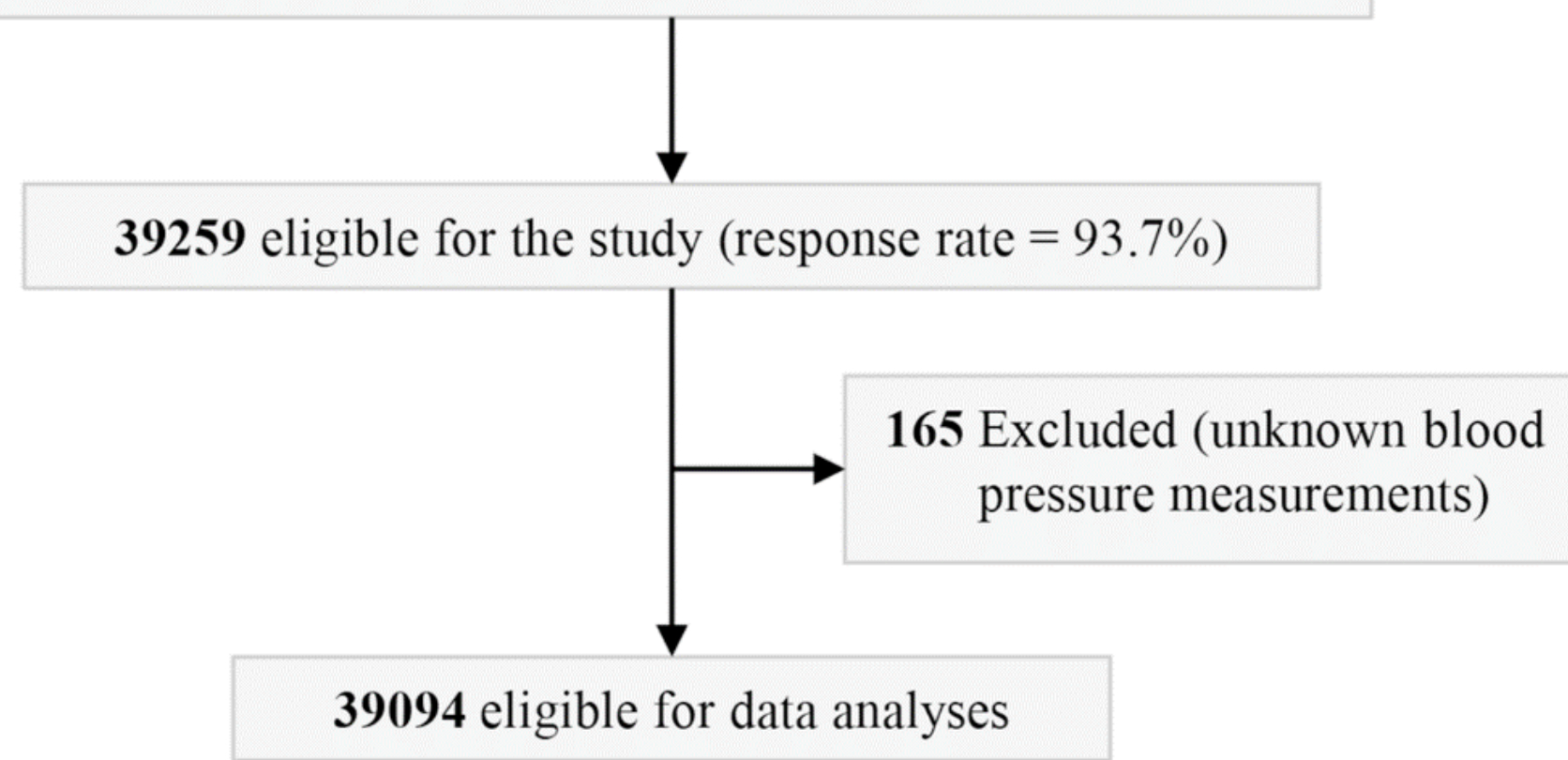

Figure 1

The flowchart of participant recruitment 


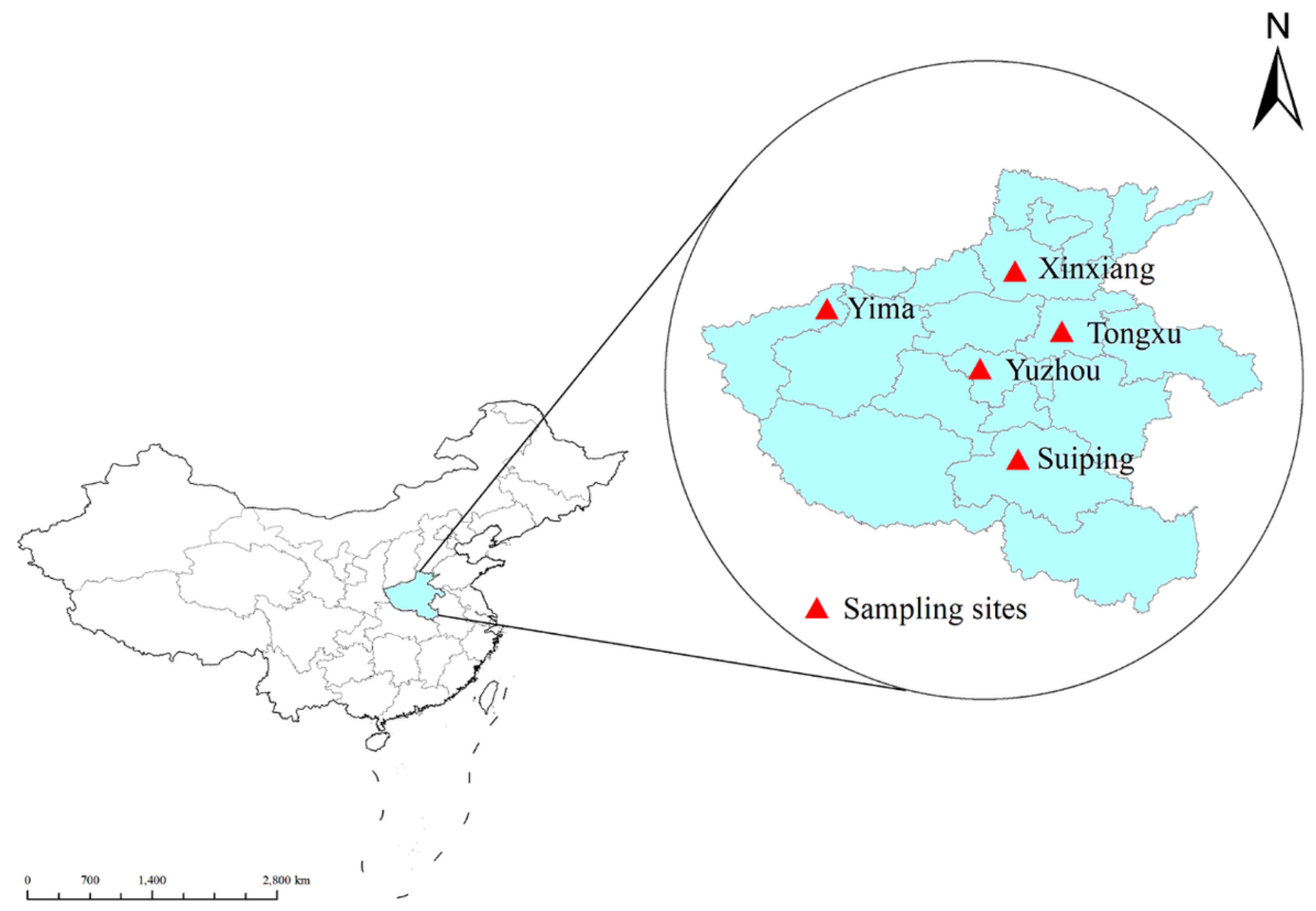

Figure 2

Locations of study regions in the study. Note: The designations employed and the presentation of the material on this map do not imply the expression of any opinion whatsoever on the part of Research Square concerning the legal status of any country, territory, city or area or of its authorities, or concerning the delimitation of its frontiers or boundaries. This map has been provided by the authors. 

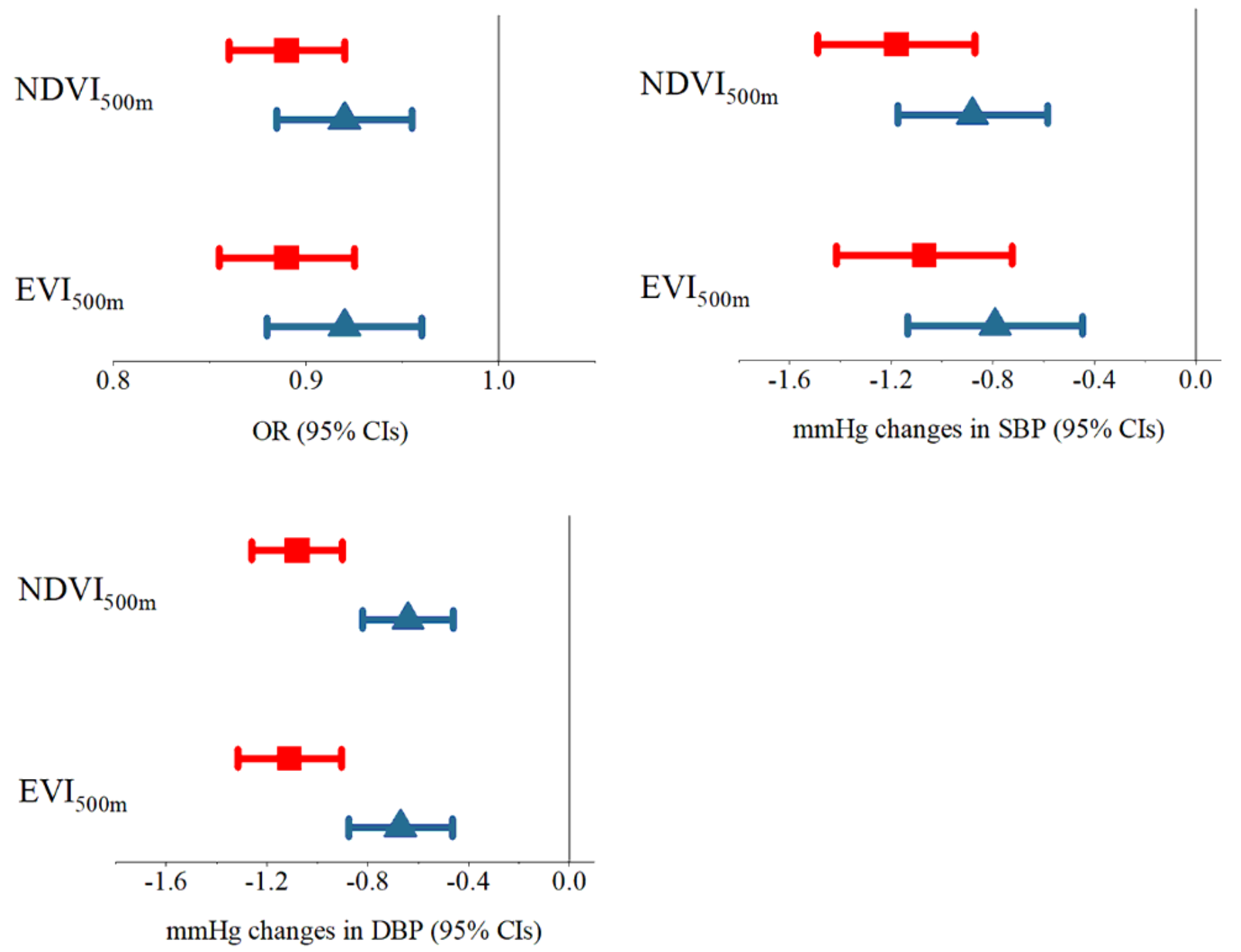

\section{Figure 3}

The relationships between residential greenness and hypertension and blood pressure. Abbreviations: $\mathrm{NDVI}$, normalized difference vegetation index; EVI, enhanced vegetation index; SBP, systolic blood pressure; DBP, diastolic blood pressure. Note: Crude model: no adjustment; Adjusted model: Adjusted for age, sex, marriage, education, income, smoking, drinking, fat-rich diet, exercise, family history of hypertension, BMI, and PM2.5. 

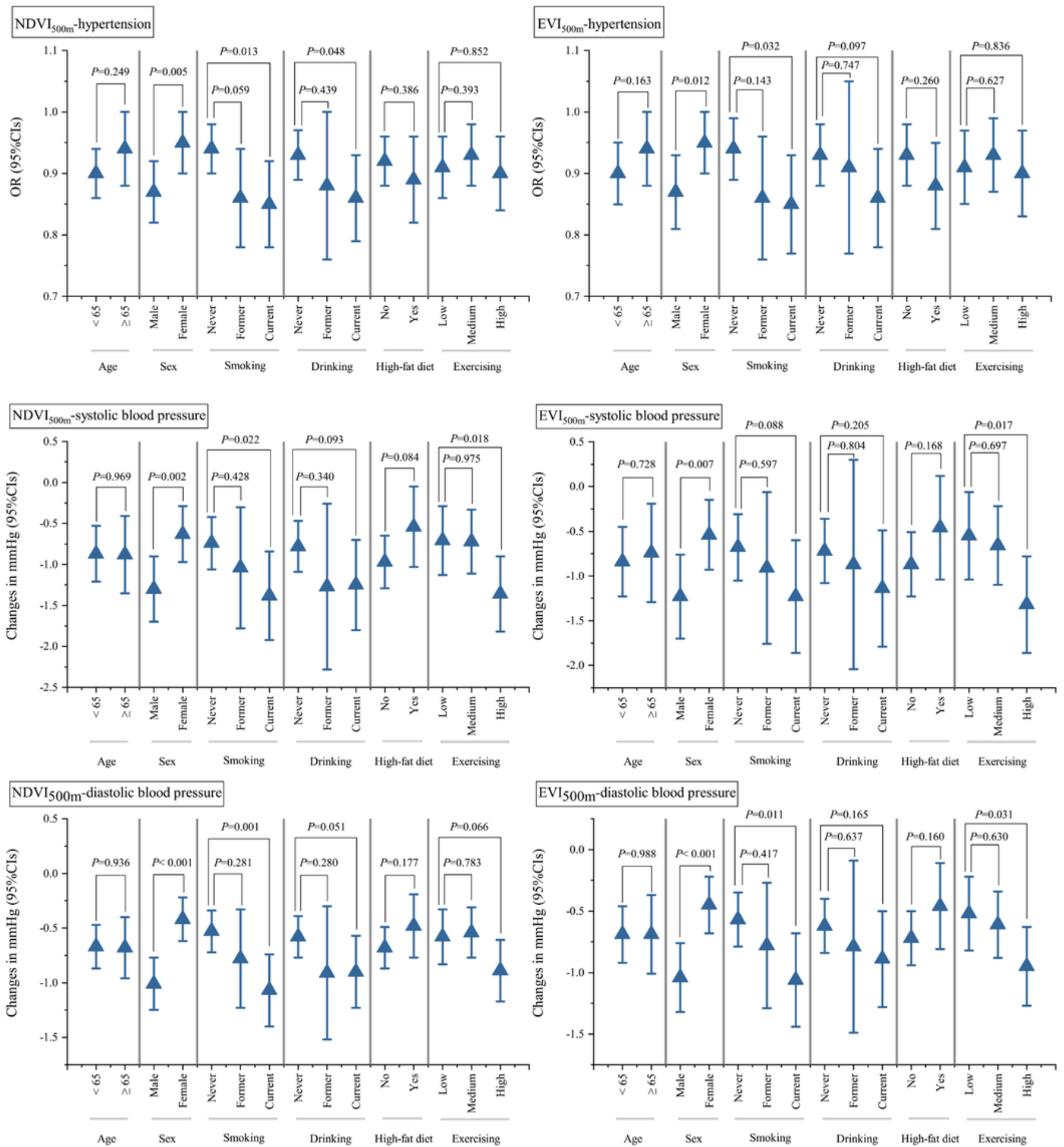

\section{Figure 4}

Interaction analyses in the relationships between residential greenness and hypertension and blood pressure. Abbreviations: NDVI, normalized difference vegetation index; EVI, enhanced vegetation index. Note: Adjusted for age, sex, marriage, education, income, smoking, drinking, fat-rich diet, exercise, family history of hypertension, BMI, and PM2.5. 


\section{Supplementary Files}

This is a list of supplementary files associated with this preprint. Click to download.

- HTNsupplement0125.docx 